\title{
Painful left thigh mass
}

\author{
Flávia A. Sakamoto • Carl S. Winalski • \\ Kevin L. Golden • John E. Ready
}

Published online: 22 May 2013

(C) ISS 2013

\begin{abstract}
Answer
Cystic echinococcosis (hydatid cyst of the femur and soft tissue).
\end{abstract}

\section{Discussion}

The patient lived alongside dogs and sheep on a rural Greek farm until he was a teenager. Seventeen years before presentation he emigrated to the United States. The radiographic findings of a lytic bone lesion with cortical destruction, scalloping, and periosteal reaction suggested a primary bone tumor (Fig. 1 in the case presentation). After MRI, chondrosarcoma and sarcoma with necrosis were suspected because of the regions of fluid-like signal intensity within the large soft tissue mass (Fig. 2 in the case presentation). Although echinococcal cyst was also considered, this diagnosis was incorrectly dismissed because the patient had not been on a farm for over 30 years. Therefore, the potential for disease spread and anaphylaxis were not considered prior to

The case presentation can be found at doi: 10.1007/s00256-013-1634-3.

F. A. Sakamoto · C. S. Winalski $(\bowtie)$

Imaging Institute, Cleveland Clinic,

9500 Euclid Avenue, A21,

Cleveland, OH 44195, USA

e-mail: winalsc@ccf.org

C. S. Winalski

Department of Biomedical Engineering, Lerner Research Institute, Cleveland Clinic,

Cleveland, OH 44195, USA

K. L. Golden

Department of Pathology, Brigham \& Women's Hospital,

Boston, MA 02115, USA

J. E. Ready

Department of Orthopaedic Surgery, Brigham \& Women's Hospital, Boston, MA 02115, USA biopsy. Percutaneous CT-guided 14-gauge needle biopsy targeted the vastus lateralis to enable needle track resection at surgery. Aspirated fluid and cyst wall fragments were diagnostic of hydatid disease (HD) from Echinococcus granulosus.

Radical resection of the soft tissue component with debridement of the femur was performed. Low power histological evaluation showed the hydatid cyst and daughter vesicles with central clear areas that had contained primarily fluid, as well as the perihydatidic adventitia (host response; Fig. 3 in the case presentation). Higher magnification demonstrated the laminated echinococcal membrane and multinucleated giant cells in the adventitia (Fig. 3 inset, in the case presentation). Three years after resection, there was local recurrence with a pathological femoral fracture. Treatment included wide excision requiring proximal femoral replacement with a megaprosthesis total hip arthroplasty. Now, 7 years later, the patient is functioning well without assistive devices and with no recurrence.

On radiographs, osseous HD may mimic an aggressive primary or secondary bone tumor, bacterial osteomyelitis, or fibrous dysplasia with coarse trabeculation, sclerosis, bone expansion, erosion, cortical destruction, and osteolysis [1, 2]. MRI may demonstrate characteristic round structures, daughter vesicles, within the mother cyst that are lower signal intensity on T1-weighted images with no enhancement after contrast medium administration, a finding helpful in the diagnosis (Fig. 2 in the case presentation) $[1,2]$. The younger daughter vesicles appear darker on T1-weighted images because they contain more pure fluid and fewer protoscoleces than the mother cyst [3]. While the "rim sign," a thick dark cyst wall on T2-weighted images, is characteristic at other sites [4], it is not as specific a finding in muscle. After contrast medium administration, the perihydatidic layer typically enhances [1] (Fig. 2c in the case presentation).

Hydatid disease, which occurs in endemic areas worldwide, has a well-described life cycle [5-7]. Dogs are 
infected when they ingest echinococcal cysts in the viscera of grazing livestock. Worms in the dog's intestine release eggs, which intermediate hosts, including humans, may ingest in contaminated food. Larvae from the hatched eggs disseminate hematogenously to various organs, and primary echinococcosis occurs when a hydatid cyst develops. The inner layer produces brood capsules and protoscoleces that may form daughter vesicles or disseminated disease, if the cyst ruptures. No perihydatidic adventitia forms in bone, and intramedullary spread readily occurs [1]. Hydatid cysts grow slowly and asymptomatically, $1-30 \mathrm{~mm} / \mathrm{year}$, generally over decades [7].

No clinical signs/symptoms, biochemical tests, or radiological signs are specific for HD [8]. A combination of imaging and serology is most reliable for diagnosis [9]. Anaphylaxis or secondary echinococcosis from lymphatic or hematogenous spread may occur if the cyst wall ruptures spontaneously or iatrogenically [10]. The risk of anaphylaxis at biopsy may be overstated; a review of 5,943 needle procedures for HD found low rates of fatal anaphylaxis $(0.03 \%)$ and reversible allergic reactions $(1.67 \%)$ [11]. If a diagnostic biopsy is performed, concomitant anthelmintic medication is recommended [10].

Skeletal HD, which is rare $(0.2 \%$ of cysts $)$ and most commonly involves the spine [1], is best treated by resection of all involved bone and soft tissue, avoiding cyst rupture; high-dose systemic anthelmintic therapy can be added [9]. Incomplete removal may result in recurrence, as demonstrated with our patient.

In conclusion, osseous echinococcosis is a rare condition that may mimic a tumor. A patient's life history must be considered when formulating a differential diagnosis and decades may have passed between the patient living in an endemic region and presentation. Recognizing characteristic daughter vesicles on imaging may suggest the diagnosis before intervention, permitting the avoidance of iatrogenic cyst rupture and use of anthelmintic medication during biopsy.

Acknowledgments We thank Danny A. Milner Jr., MD for his help with the pathology and manuscript review. We appreciate the assistance provided by Ms. Megan Griffiths, scientific writer for the Cleveland Clinic Imaging Institute, in the preparation of the manuscript.
Conflict of interest The authors declare that they have no conflict of interest.

\section{References}

1. Arkun R, Mete BD. Musculoskeletal hydatid disease. Semin Musculoskelet Radiol. 2011;15(5):527-40.

2. Pedrosa I, Saiz A, Arrazola J, Ferreiros J, Pedrosa CS. Hydatid disease: radiologic and pathologic features and complications. Radiographics. 2000;20(3):795-817.

3. von Sinner WN, Rifai A, te Strake L, Sieck J. Magnetic resonance imaging of thoracic hydatid disease. Correlation with clinical findings, radiography, ultrasonography, CT and pathology. Acta Radiol. 1990;31(1):59-62.

4. Taourel P, Marty-Ane B, Charasset S, Mattei M, Devred P, Bruel JM. Hydatid cyst of the liver: comparison of CT and MRI. J Comput Assist Tomogr. 1993;17(1):80-5.

5. Thompson RCA, McManus DP. Aetiology: parasites and lifecycles. In: Eckert J, Gemmell MA, Meslin F-X, Pawłowski ZS, editors. WHO/OIE manual on echinococcosis in humans and animals: a public health problem of global concern. Paris: World Health Organization and World Organisation for Animal Health; 2001. p. 1-19.

6. McManus DP, Zhang W, Li J, Bartley PB. Echinococcosis. Lancet. 2003;362(9392):1295-304.

7. da Silva AM. Hydatid cyst/cystic echinococcosis: anatomical and surgical nomenclature and method to quantify the cyst content solidification. Chin Med J. 2011;124(18):2806-12.

8. Barnes TS, Deplazes P, Gottstein B, Jenkins DJ, Mathis A, SilesLucas $M$, et al. Challenges for diagnosis and control of cystic hydatid disease. Acta Trop. 2012;123(1):1-7.

9. Brunetti E, Kern P, Vuitton DA. Expert consensus for the diagnosis and treatment of cystic and alveolar echinococcosis in humans. Acta Trop. 2010;114(1):1-16.

10. Pawłowski ZS, Eckert J, Vuitton DA, Ammamm RW, Kern P, Craig PS, et al. Echinococcosis in humans: clinical aspects, diagnosis and treatment. In: Eckert J, Gemmell MA, Meslin F-X, Pawłowski ZS, editors. WHO/OIE manual of Echinococcosis in humans and animals: a public health problem of global concern. Paris: World Health Organization and World Organisation of Animal Health; 2001. p. 20-72.

11. Neumayr A, Troia G, de Bernardis C, Tamarozzi F, Goblirsch $\mathrm{S}$, Piccoli L, et al. Justified concern or exaggerated fear: the risk of anaphylaxis in percutaneous treatment of cystic echinococcosis-a systematic literature review. PLoS Negl Trop Dis. 2011;5(6):e1154. 